\title{
Reasons for Polytobacco Use among Young Adults: Scale Development and Validation
}

\author{
Corla J. Berg ${ }^{1}$, Regine Hoardörfer ${ }^{1}$, Gillion Schauer ${ }^{2}$, Getochew Betelihem ${ }^{1}$, Matthew Masters ${ }^{1}$, Bennett McDonald ${ }^{3}, J_{i l l}$ Shah ${ }^{4}$, \\ Jackelyn Payne ${ }^{1}$, Raina Sarmah ${ }^{1}$,Matthew Thomas ${ }^{5}$,Michoel Windle ${ }^{1}$
}

\begin{abstract}
INTRODUCTION Limited research has examined reasons for polytobacco use, an increasing public health problem, particularly among young adults. We examined reasons for polytobacco use among users of more than one tobacco product in the past 4 months enrolled in an ongoing sixwave longitudinal study of 3,418 students aged 18-25 from seven US colleges and universities. METHODs An expert panel generated items related to reasons for polytobacco use, included in Wave 3 (administered in Summer 2015). Participants reporting use of more than one tobacco product in the past four months $(n=540)$ were asked to complete the Reasons for Polytobacco Use scale and measures related to tobacco/nicotine use/dependence, use motives, perceptions of tobacco, parental/friend use, other substance use, and mental health. We conducted a factor analysis and then examined convergent and discriminant validity for the derived factors.

RESULTS Our sample was an average age of 20.40 ( $\mathrm{SD}=1.84), 48.0 \%$ male, and $21.9 \%$ Black. Four factors were identified: Instrumentality, Social Context, Displacement, and Experimentation. Instrumentality was the only factor associated with little cigar/cigarillo and marijuana use. Displacement and Social Context showed similar associations; however, Social Context was associated with having friends who used tobacco while Displacement was not. Experimentation was associated with greater perceived addictiveness and harm of using tobacco products as well as greater perceived social acceptability of tobacco use.

concLusions Each of the four factors identified demonstrated unique convergent and discriminant validity. The use of this scale to characterize polytobacco using young adults may help inform and target cessation or prevention interventions.
\end{abstract}

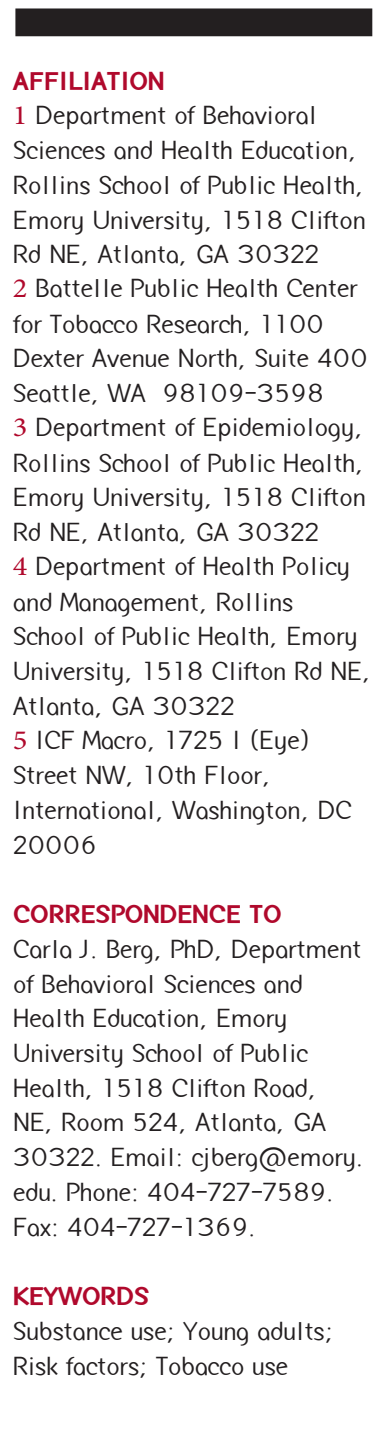

\section{INTRODUCTION}

While traditional cigarettes continue to be the main source of tobacco use in the US, ${ }^{1,2}$ various alternative tobacco products (ATPs), including little cigars and cigarillos (LCCs), smokeless tobacco (SLT), electronic cigarettes (e-cigarettes), and hookah, have recently been introduced to the US market, with use and awareness of these products dramatically increasing, particularly among young adults ${ }^{3-5}$. ATP use represents health risks. For example, LCCs can deliver sufficient amounts of nicotine to maintain dependence and can cause several chronic diseases (e.g., coronary heart disease, lung diseases, cancer $)^{6}$. Additionally, although e-cigarettes represent promise for harm reduction in smokers, ${ }^{7-11}$ research has documented that e-liquids contain detectable levels of carcinogens (formaldehyde, certain tobacco-specific nitrosamines) and toxins (diethylene glycol), ${ }^{12,13}$ and e-cigarette use has adverse pulmonary effects ${ }^{14}$. Furthermore, hookah use produces carbon monoxide, nicotine, tar, and heavy metals at levels similar to or higher than cigarettes ${ }^{3}$.

ATPs have significantly altered the terrain of tobacco use, particularly among young adults (i.e., those aged 18-24 years). Per the 2012-2013 National Adult Tobacco Survey, current 
use prevalence in this population was: $18.5 \%$ cigarettes, $3.4 \%$ LCCs, $4.4 \%$ SLT, $2.4 \%$ e-cigarettes, and $2.5 \%$ hookah $^{15}$. Of particular relevance to the current study, recent research has documented high rates of polytobacco use, particularly in this population ${ }^{16-18}$. Roughly $15-30 \%$ of young adult smokers currently use more than one tobacco product; ${ }^{19,20}$ among ATP users, polytobacco use has increased to $40-50 \%^{21,22}$. Beyond the risks of using a single ATP, polytobacco use may increase risk of nicotine dependence $\mathrm{e}^{23-25}$.

The reasons for polytobacco use are not well known. One major reason for using various substances concurrently may be to achieve the synergistic effects of substances used simultaneously ${ }^{26,27}$. This may hold true both within the tobacco product category and outside the range of tobacco products (e.g., marijuana) ${ }^{28}$. Another possible reason for polytobacco use may be that some are more socially appealing than others. For example, hookah use is seen as particularly socially acceptable, ${ }^{29-31}$ whereas other products such as cigarettes are not ${ }^{32}$. Thus, people may prefer the use of one tobacco product over another in certain social settings. Additionally, some tobacco products might be used to reduce or quit the use of another or to circumvent smoke-free policies ${ }^{33}$. Finally, experimentation is another possible reason for polytobacco use, particularly among young adults ${ }^{34}$. Notably, many ATPs, particularly e-cigarettes and hookah, are often perceived as less harmful and addictive among young adults, thus increasing the likelihood of experimentation and uptake $\mathrm{e}^{32}$.

Drawing from the Theory of Planned Behavior ${ }^{35,36}$ and Social Cognitive Theory ${ }^{37}$, several individual and sociocontextual characteristics may be distinctly associated with different reasons for polytobacco use. For example, more favorable attitudes toward use or lower perceived risk of tobacco use may be related to polytobacco use for purposes of achieving synergistic effects, displacement, or experimentation. Moreover, social environment and subjective norms may play a role in polytobacco use. Those with parents, friends, and other social influences who use tobacco may perceive social norms that are more conducive to tobacco use and may be more sensitive to social context ${ }^{32,38}$. New tobacco products may also be more available to them for experimentation ${ }^{32}$, 38. Additionally, polytobacco use might be associated with polysubstance use and a genetic propensity for addiction in general, particularly if a main reason for polytobacco use is to achieve the synergistic effects of substances ${ }^{32,38}$. Furthermore, individual mental health may differentially impact reasons for polytobacco use. For example, depressive symptoms may be associated with polytobacco use among heavier users who may be self-medicating. Depressive symptoms, however, may not be associated with experimentation ${ }^{38-41}$. Finally, certain reasons for polytobacco use might be associated with use of specific tobacco or nicotine products or with higher levels of addiction.

In summary, there has been an increase in polytobacco use, but limited research, particularly quantitative research, has been conducted to assess the context and reasons for polytobacco use. Assessing the reasons for polytobacco use and examining correlates of these reasons may inform intervention efforts. Thus, the current study aimed to develop and test the reliability and validity of a scale to assess reasons for polytobacco use among young adults reporting the use of more than one tobacco or nicotine product in the past four months.

\section{METHODS}

\section{Participants and Procedures}

The parent study, entitled Project DECOY (Documenting Experiences with Cigarettes and Other Tobacco in Young Adults), was approved by the Emory University and ICF International Institutional Review Boards as well as those of the participating colleges. Project DECOY is a sequential mixed-methods ${ }^{42}$ longitudinal panel study of 3,418 college students from seven colleges in Georgia. Note that, while recent estimates of use prevalence of LCCs, e-cigarettes, and hookah among young adults in Georgia are not available, 2014 CDC Behavioral Risk Factor Surveillance data ${ }^{43}$ indicated a prevalence of current cigarette and SLT use of $16.4 \%$ and $5.4 \%$, respectively. The colleges in this study included two public universities/colleges, two private universities, two community/ technical colleges, and a historically black university located in rural and urban settings. Detailed information on this study is provided elsewhere ${ }^{44}$ but briefly summarized here.

Contact information (e.g., email addresses) was obtained from the registrar's office from each college/university for students meeting eligibility criteria (i.e., ages $18-25$; able to speak English). Three thousand randomly selected 18-25 year olds were selected from one private and two public universities. The remainder of the schools had 18-25 year old student populations of less than 3,000; thus, the entire student population of that age range at those schools was included in recruitment. Response rates ranged from $15.4 \%$ to $27.6 \%$ at the technical colleges; $12.0 \%$ and $19.2 \%$ at the public colleges/ universities; $18.8 \%$ and $59.4 \%$ at the private universities; and $23.1 \%$ at the historically black university. Our overall response rate of $22.9 \%(\mathrm{~N}=3,574 / 15,607)$, albeit low, was over a very short time frame ( 24 hours at the private schools to seven days at the technical colleges) and met our sampling quota targets ${ }^{44}$. Our intent was to enroll participants who were engaged in email and were potentially more likely to be retained in the 
subsequent waves of the larger, multi-wave longitudinal project. The sociodemographics characteristics of the baseline sample was largely reflective of the student bodies of the college campuses included in this study; however, our sample was disproportionately female ${ }^{44}$.

Data collection began in Fall 2014 and consists of selfreport assessments via an online survey every four months for two years (during Fall, Spring, and Summer). Current analyses draw from the baseline assessment of sociodemographic information and Wave 3 data collected in Summer 2015. Retention at Wave 3 was $83.9 \%(\mathrm{n}=2869 / 3418)$. In order to ensure that we had sufficient sample size, we directed all participants who used more than one tobacco product in the past 4 months (a time frame selected to cover the period of time between assessments) to the Reasons for Polytobacco Use section. At Wave 3, 1,087 (37.9\%) of participants used any tobacco in the past 4 months (786 [27.4\%] of all participants used any tobacco in the past 30 days). For the current analysis, we included the 540 participants who used more than one tobacco product in the past 4 months $(18.8 \%$ of the total; $49.7 \%$ of tobacco users). Notably, 221 (40.9\% of polytobacco users) used two tobacco products, 189 (35.0\%) used three, and $130(24.1 \%)$ used four or more.

\section{Measures}

Data collected at Wave 3 included a range of psychosocial and substance use variables. Below we outline our primary measure of focus - the newly developed Reasons for Polytobacco Use Scale - and the correlates of interest.

Reasons for Polytobacco Use. After reviewing the literature, an expert panel including the authors of the current paper and colleagues in the area of tobacco use, particularly ATP use, developed a list of potential reasons for polytobacco use (Table 2). Participants were asked, "How true for you is each possible reason for using more than one tobacco product in the past 4 months?" with response options of $0=$ not at all true to $6=$ extremely true.

Sociodemographics. We assessed age, sex, race, and ethnicity.

Tobacco Use. Participants were first asked to report the number of days they used each tobacco product - cigarettes; little cigars or cigarillos (such as Swisher Sweets or Black n Milds); smokeless tobacco (such as chew, snus, or dissolvable tobacco); e-cigarettes (such as Blu or Njoy); hookah - in the past 4 months (to cover the duration of time between each wave of assessment). Those that reported any use in the past 4 months were then asked to report the number of days they used the respective tobacco product. Pictures were presented alongside each to aid in participant understanding. Tobacco use was dichotomized as any versus no use in the past 30 days, as preliminary analyses indicated similar results using these as continuous variables.

The Hooked on Nicotine Checklist. The Hooked on Nicotine Checklist ${ }^{45,46}$ is a reliable and valid measure of diminished autonomy over tobacco. It is uniquely suited for use with smokers whose cigarette consumption is low. The verbiage of this scale was adapted in the current study to be applicable to all types of tobacco and nicotine product use (i.e., not only cigarette smoking; e.g., "Have you ever tried to quit using tobacco or nicotine, but couldn't?"). Cronbach's alpha for this scale in this study was .94 .

The Motives for Smoking Scale. The Motives for Smoking Scale ${ }^{47,48}$ assesses the extent to which each of 15 smokingrelated motives is true for a participant ( $1=$ not at all true to $5=$ very true). The measure contains questions about four common motives: social (4 items, e.g., "Smoking helps you fit in with other people"), self-confidence ( 4 items, e.g., "Smoking makes you feel more self-confident"), boredom relief (2 items, e.g., "Smoking is something to do when you're bored"), and affect regulation (5 items, e.g., "Smoking helps you calm down when you're feeling tense or nervous," "Smoking cheers you up when you're in a bad mood"). Higher scores indicate that the motive is more relevant. The verbiage of this scale was adapted in the current study to be applicable to all types of tobacco and nicotine product use (e.g., "Using tobacco or nicotine helps you fit in with other people"). In the current study, alphas for the social subscale, the self-confidence subscale, the boredom relief subscale, and the affect regulation subscale were.89, .86 , .94 , and .92 , respectively.

Perceptions of Tobacco Products. We also asked about perceptions of tobacco products (cigarettes, LCCs, SLT, e-cigarettes, hookah) on a Likert scale of $1=$ not at all to $7=$ extremely. This included perceptions about addictiveness of each tobacco product, harmfulness of product use, and social acceptability of each product ${ }^{32}$.

Readiness to Quit. Among past 4-month users of any tobacco product, participants were asked, "What best describes your intentions regarding quitting the use of all nicotine and tobacco: never expect to quit; may quit in the future, but not in the next 6 months; will quit in the next 6 months; and will quit in the next month" ${ }^{\prime 9}$. For the present study, this variable was dichotomized as intending to quit all tobacco products in the next 30 days versus all other responses.

Social Factors. We asked if a parent currently used each tobacco product ${ }^{32}$ and the number of five closest friends using each tobacco product ${ }^{32}$. These items were operationalized as 
dichotomous variables (e.g., at least one friend used versus none).

Other Substance Use. Participants were first asked to report the number of days they used alcohol and marijuana, respectively. Those that reported any use in the past 4 months were then asked to report the number of days they used the respective product. Given the distributions of use, alcohol use was used as a continuous variable, while marijuana use was used as a dichotomous variable.

Depressive Symptoms. We assessed depressive symptoms using the Patient Health Questionnaire - 9 item (PHQ-9) $)^{50}$. We developed a continuous variable resulting from a sum of the items. Cronbach's alpha in the current study was .86 .

\section{Data Analysis}

We conducted a factor analysis of the Reasons for Polytobacco Use items using Promax rotation. We used eigenvalues of greater than 1 as the criteria for number of factors. Then, we examined the content and internal consistency of the factors. Descriptive statistics regarding sociodemographics, tobacco use characteristics, and factors aimed at examining convergent and discriminant validity were calculated. We then conducted bivariate analyses examining subscale scores in relation to these correlates of interest. Analyses were conducted in SPSS 23.0, and alpha was set at .05.

\section{RESULTS}

\section{Participont Characteristics}

Our sample was an average age of 20.40 ( $\mathrm{SD}=1.84$ ), 48.0\% male, 64.8\% White, 21.9\% Black, and 8.1\% Hispanic (Table 1). Our sample included 40.9\% cigarette users, 30.4\% LCC users, 13.5\% SLT users, 31.3\% e-cigarette users, and 30.7\% hookah users. On average, participants used $2.47(\mathrm{SD}=1.24)$ types of tobacco products in the past 30 days. Average number of days of alcohol use was 6.53 ( $\mathrm{SD}=6.82$ ), and $33.0 \%$ of our sample reported using marijuana in the past 30 days.

\section{Factor Analysis}

Factor analysis identified four factors (see Table 2): 1) Instrumentality, which indicated a range of functions polytobacco use served related to physical sensations (e.g., buzz, enhancing effects of other tobacco products or other substances), appetite reduction, and other substance use reduction; 2) Social Context, indicating use of different products in different social contexts or in relation to self-presentation; 3) Displacement, indicating use of one tobacco product to reduce or quit the use of another or using one tobacco product to circumvent smoke-free policies; and 4) Experimentation, indicating experimental use of tobacco products but no specific
Table 1. Participant sociodemographic and tobacco use characteristics, $n=540$

\begin{tabular}{lc} 
Variable & M (SD) or N $(\%)$ \\
Age (SD) & Sociodemographics \\
\multicolumn{2}{c}{$20.40(1.84)$} \\
Female & $281(52.0)$ \\
Male & $259(48.0)$ \\
& Race/Ethnicity $(\%)$ \\
White & $350(64.8)$ \\
Black & $118(21.9)$ \\
Asian & $31(5.7)$ \\
Other & $41(7.6)$ \\
Hispanic (\%) & $44(8.1)$ \\
& $174(32.2)$ \\
Public university & $190(35.2)$ \\
Private college/university & $69(12.8)$ \\
HBCU & $107(19.8)$ \\
Technical college & School Type
\end{tabular}

\section{Tobacco-Related Measures}

Current tobacco use (\%)

$\begin{array}{ll}\text { Cigarettes } & 221(40.9) \\ \text { LCCs } & 164(30.4) \\ \text { SLT } & 73(13.5) \\ \text { E-cigarettes } & 169(31.3) \\ \text { Hookah } & 166(30.7) \\ \text { Number of products used (SD) } & 2.47(1.24) \\ \text { Nicotine dependence a (SD) } & 2.06(3.21) \\ \text { Social motives b (SD) } & 7.15(3.56) \\ \text { Self-enhancement motives b (SD) } & 6.23(3.33) \\ \text { Boredom motives b (SD) } & 3.84(2.40) \\ \text { Affect regulation motives b (SD) } & 10.25(5.69)\end{array}$

Social Factors

Parental use of tobacco (\%) 209 (38.7)

Friend use of tobacco (\%)

$491(90.9)$

Substance Use \& Mental Health

Number of days alcohol use, past $30 \quad 6.53$ (6.82) days (SD)

Any marijuana use, past 30 days (\%) 178 (33.0)

Depressive symptoms c (SD) $16.91(90.02)$

a Per the Hooked on Nicotine Checklist; b Per the Motives for Smoking Scale; c Per the PHQ-9

Note: Perceived addictiveness, harm to health, and social acceptability not reported in tables, as this includes 15 variables ( 3 dimensions $\times 5$ tobacco products).

Abbreviations: $\mathrm{HBCU}=$ Historically Black College or University; $\mathrm{LCC}=$ little cigars or cigarillos; SLT = smokeless tobacco. 
link between the use of the distinct products. Two items were deleted (My friends or family introduced me to a new tobacco product; I use different tobacco products for different reasons, e.g., to concentrate, to relax), as these items were deemed vague and did not assess specific reasons for use. These four factors accounted for $62.5 \%$ of the variance. Cronbach's alphas for each subscale were: $.91, .78, .82$, and .56 , respectively. The correlations ranged from .14 between Displacement and Experimentation to .58 between Instrumentality and Displacement (Table 3).

\section{Convergent and Discriminant Validity}

Table 3 provides data regarding convergent and discriminant validity. Instrumentality was associated with current use of each tobacco product; greater number of tobacco products used; higher nicotine dependence; higher motivation subscale scores across motivations (e.g., social, self-enhancement, boredom, affect regulation); friends' tobacco use; current marijuana use (but not alcohol use); greater perceived addictiveness of cigarettes ( $\mathrm{r}=-.09, \mathrm{p}=.033$, not in tables $)$; and greater perceived harm to health of cigarette use $(r=-.09, p=.029)$. Social

Table 2. Reasons for Polytobacco Use Scale items and factor loadings

\section{Component}

1

Instrumentality

Using different products together provides a great buzz.

I get a different kind of buzz from different tobacco products.

I get sick of the taste of some tobacco products but enjoy the buzz, so I use other tobacco products sometimes.

I use some tobacco products to increase the effects of other tobacco products.

I use some tobacco products to increase the effects of alcohol.

I use some tobacco products to increase the effects of illicit drugs.

I use some tobacco products to increase the effects of prescription drugs.

I was using more than one tobacco product to help me reduce my appetite.

I was using more than one tobacco product to help me reduce my use of alcohol.

I was using more than one tobacco product to help me reduce my use of other drugs.

\section{Social Context}

I use different tobacco products around different people (friends, peers at school, family).

I use different tobacco products in different places (home, school, work, bars, parties).

I use tobacco product others are using to be sociable.

I'm ok with the image of me that using some tobacco products reflects, but not others.

\section{Displacement}

I regularly use one tobacco product but only experimented with the other tobacco products.

I was using one tobacco product to try to quit using another tobacco product.

I was using one tobacco product to try to reduce my use of other tobacco products.

I was using more than one tobacco product because I couldn't smoke (cigarettes, cigars, etc.) in some places or at some times.

\section{Experimentation}

The use of one tobacco product had nothing to do with the use of the other.

I just like to experiment with different tobacco products.

I only experimented with these products but did not use any of them regularly.

\begin{tabular}{|c|c|c|c|}
\hline .844 & 111 & -.136 & .047 \\
\hline .478 & .302 & -.016 & .095 \\
\hline .626 & .162 & .027 & -.007 \\
\hline .881 & -.021 & -.009 & -.059 \\
\hline .524 & .412 & -.239 & -.034 \\
\hline .712 & .233 & -.201 & -.060 \\
\hline .794 & -.083 & .117 & -.033 \\
\hline .677 & -.128 & .278 & .007 \\
\hline .786 & -.156 & .223 & -.026 \\
\hline .827 & -.149 & .158 & .039 \\
\hline-.125 & .891 & .081 & -.115 \\
\hline-.030 & .869 & .103 & -.133 \\
\hline .126 & .593 & -.038 & .122 \\
\hline .178 & .403 & .049 & .159 \\
\hline-.147 & .335 & .400 & .281 \\
\hline .044 & .023 & .874 & -.058 \\
\hline .010 & .026 & .917 & -.066 \\
\hline .224 & .134 & .583 & -.017 \\
\hline-.156 & .076 & -.053 & .772 \\
\hline .128 & .045 & .166 & .589 \\
\hline .044 & -.189 & -.148 & .811 \\
\hline
\end{tabular}

Note: Extraction Method: Principal Component Analysis. Rotation Method: Promax with Kaiser Normalization. Rotation converged in 6 iterations.

Note: Cronbach's alpha for Instrumentality, Social Context, Displacement, and Experimentation were: 90, .91, .78, .77, and .56, respectively. Average subscale scores were: Instrumentality 1.54 ( $S D=0.87)$; Social Context 2.00 ( $S D=1.15)$; Displacement 1.69 ( $S D=1.04)$; and Experimentation $2.87(S D=1.41)$. 
Table 3. Correlations among Reasons for Polytobacco Use subscales and related factors

\begin{tabular}{|c|c|c|c|}
\hline Variable & Instrumentality & Social Contert & Displacement \\
\hline & \multicolumn{3}{|c|}{ Reasons for Polytobacco Use } \\
\hline Instrumentality & -- & $.53^{* * *}$ & $.58^{* * *}$ \\
\hline Social Context & & -- & $38^{* * *}$ \\
\hline Displacement & & & -- \\
\hline
\end{tabular}

Tobacco-Related Measures

Current Tobacco Use

\begin{tabular}{|c|c|c|c|}
\hline Cigarettes $\neq$ & $.10 *$ & $.10^{*}$ & $.30 * * *$ \\
\hline LCCs $\neq$ & $.12^{* *}$ & .03 & .05 \\
\hline SLT $\neq$ & $.10^{*}$ & $.13^{* *}$ & $.15^{* *}$ \\
\hline E-cigarettes $\neq$ & $.10^{*}$ & $.13^{* *}$ & $.19^{* * *}$ \\
\hline Hookah $\neq$ & $.18^{* * *}$ & $.11^{*}$ & $.10^{*}$ \\
\hline Number of products used & $.22^{* * *}$ & $.18^{* * *}$ & $.29^{* * *}$ \\
\hline Nicotine dependence ${ }^{a}$ & $.18^{* *}$ & $.09^{*}$ & $.46^{* * *}$ \\
\hline Social motives ${ }^{b}$ & $.42^{* * *}$ & $.44^{* * *}$ & $38^{* * *}$ \\
\hline Self-enhancement motives ${ }^{b}$ & $.51^{* * *}$ & $.39^{* * *}$ & $.48^{* * *}$ \\
\hline Boredom motives ${ }^{b}$ & $39^{* * *}$ & $.32^{* * *}$ & $.48^{* * *}$ \\
\hline Affect regulation motives ${ }^{b}$ & $40^{* * *}$ & $.28^{* *}$ & $49^{* * *}$ \\
\hline Readiness to quit all tobacco $\neq$ & .04 & -.02 & -.01 \\
\hline
\end{tabular}

Readiness to quit all tobacco $\neq$

Social Factors

Parental use of tobacco $\neq$

$-.04$

$-.03$

01

Friend use of tobacco $\neq$

$.11^{*}$

$.09^{* *}$

.06

Substance Use \& Mental Health

Number of days of alcohol use, past 30 days

Any marijuana use, past 30 days $\neq$

Context was associated with current use of cigarettes, SLT, e-cigarettes, and hookah (but not LCCs); greater number of tobacco products used; higher nicotine dependence; higher motivation subscale scores across motivations; friends' tobacco use; number of days of alcohol use in the past month (but not marijuana); and greater perceived social acceptability of hookah use $(\mathrm{r}=.11, \mathrm{p}=.008)$. Displacement was associated with current use of cigarettes, SLT, e-cigarettes, and hookah (but not LCCs); greater number of tobacco products used; higher nicotine dependence; higher motivation subscale scores across motivations; alcohol use (but not marijuana); greater perceived harm to health of e-cigarette use $(\mathrm{r}=-.09, \mathrm{p}=.039)$; and greater perceived social acceptability of cigarette and e-cigarette use $(\mathrm{r}=.10, \mathrm{p}=.025$ and $\mathrm{r}=.13, \mathrm{p}=.002$, respectively). Experimentation was associated with current use of e-cigarettes (but no other tobacco product); higher motivation subscale scores across motivations; friends' tobacco use; alcohol use (but not marijuana); greater perceived addictiveness of cigarettes, LCCs, SLT, and e-cigarettes ( $r$ 's ranging from .10 for e-cigarettes to .19 for cigarettes, $p$ 's<.05); greater perceived harm to health of cigarettes, LCCs, and SLT (r's ranging from .13 for e-cigarettes to .22 for cigarettes, p's $<.01$ ); and greater perceived social acceptability of use of each product (r's ranging from .10 for SLT to .21 for hookah, p's<.05).

Most noteworthy are the associations or lack of associations that discriminate the subscales. Experimentation was not 
associated with current use of any tobacco product with the exception of e-cigarettes, nor was it associated with nicotine dependence; however, Experimentation was associated with greater perceived addictiveness and harm of using tobacco products as well as greater perceived social acceptability of tobacco use. Displacement and Social Context showed similar associations with the exception that Social Context was associated with having friends who used tobacco while Displacement was not. Finally, Instrumentality and Social Context were quite similar with the exception of Instrumentality not being associated with alcohol use. Interestingly, Instrumentality was the only factor associated with current marijuana use. It is also noteworthy that sociodemographics, readiness to quit using tobacco and nicotine, parental use of tobacco, and depressive symptoms were not significantly associated with scores on any of the Reasons for Polytobacco Use Scale subscales.

\section{DISCUSSION}

There is a dearth of research, particularly quantitative research, on reasons for polytobacco use patterns among young adults. This is critical, as using tobacco during this developmental period may lead to establishment of chronic tobacco use and addiction, ultimately increasing the risk of various diseases and illnesses. Interventions aimed at addressing tobacco use among young adults must be informed by a solid understanding of different use profiles and the reasons young adults use tobacco and engage in polytobacco use. This is particularly important in this population given the significant proportion of young adult tobacco users who are polytobacco users ${ }^{16,17}$. At Wave 3 , nearly half of all tobacco users used more than one tobacco product in the past 4 months, with over half of polytobacco users using 3 or more different tobacco products. Thus, polytobacco use is a significant issue to address within this population. This study provides a preliminary scale that could be used to assess reasons for polytobacco use.

Aligned with the Theory of Planned Behavior ${ }^{35,36}$ and Social Cognitive Theory ${ }^{37}$, this study indicated that four categories of reasons for polytobacco use were identified: Instrumentality, Social Context, Displacement, and Experimentation. Instrumentality and Displacement largely reflected outcome expectancies and attitudes towards tobacco use, Social Context reflected the importance of social influences and image maintenance, and Experimentation seemed to reflect both attitudes toward tobacco products as well as social influences. Each of the subscales was correlated with one another, with the weakest correlations being found between Experimentation and each of the others, particularly Displacement. This indicates, as we might expect, that Experimentation as a reason for polytobacco use is highly distinct from the other reasons. This is further indicated by the fact that Experimentation demonstrated the most unique profile in terms of convergent and discriminant validity. For example, Experimentation was only associated with the use of e-cigarettes but not the other products, nor was it associated with symptoms of nicotine dependence. While Experimentation was associated with each of the motivations indicated by the Motives for Smoking Scale (e.g., social, self-enhancement, boredom, affect regulation), it had the lowest correlations with each among all of the subscales of the Reasons for Polytobacco Use Scale. In addition, higher Experimentation scores was associated with perceiving the various tobacco products to be more addictive and harmful to one's health, with only a couple of correlations between these measures and other subscales. Collectively, these findings might suggest that those polytobacco users who endorse using for experimentation are less motivated by social, self-enhancement, boredom, or affect regulation and are aware of the risks of tobacco use. Interestingly, Experimentation was also associated with greater perceived social acceptability, as well as having friends who use tobacco. Thus, their social context might be playing a role in their perceptions about tobacco use as well as be a source of tobacco products with which to experiment ${ }^{32,38}$.

Social Context and Displacement were correlated with one another. Accordingly, their associations with many factors were quite similar. Displacement had the highest correlations with use for boredom and affect regulation per the Motives for Smoking Scale, while Social Context had the highest correlation with using for social reasons. Moreover, Social Context was associated with having friends who use tobacco and greater perceived social acceptability of hookah use (which aligns with prior research ${ }^{32,38}$ ), while Displacement was associated with greater perceived social acceptability of cigarette and e-cigarette use. This is intuitive, particularly given that the correlation between Displacement and nicotine dependence was much higher than the correlation between Social Context and nicotine dependence scores. This might suggest that those endorsing use dictated by Social Context might have a more malleable use pattern that does not require them to substitute products when they cannot use tobacco or in efforts to quit or reduce the use of one tobacco product. On the other hand, those endorsing Displacement-related reasons may be dealing with addiction through substituting one product for another or by using one product to quit or reduce use. These are commonly endorsed reasons for switching products or using products simultaneously ${ }^{33,34}$. This aligns with the finding that perceived harm of e-cigarette use was associated with higher 
Displacement scores. Also note that cigarette use prevalence - as well as prevalence of SLT and e-cigarette use (the ATPs most likely used to displace cigarette use) - was most highly correlated with Displacement.

Finally, Instrumentality was correlated highly with both Social Context and Displacement, indicating that those endorsing these other two reason categories also used various tobacco products to achieve certain outcomes. Thus, Instrumentality demonstrated similar associations with other measures as Social Context and Displacement. However, Instrumentality was the only factor associated with current marijuana use and with current use of LCCs. This might suggest a specific profile of young adults who are likely concurrently using tobacco products and marijuana, as LCC and marijuana co-use is a use profile frequently identified among young adults ${ }^{16,51,52}$. This use profile seems to indicate using tobacco products to achieve certain outcomes - achieving a buzz, increasing the effects of one another and other drugs (particularly marijuana), or substituting tobacco for alcohol and other drugs. Relatedly, Instrumentality had the highest correlation with using for self-enhancement and also was the only factor associated with lower perceived addictiveness and harm of cigarettes. Thus, those endorsing Instrumentality may be at particularly high risk for continued tobacco use and may develop greater nicotine addiction.

It is noteworthy that parental use of tobacco, depressive symptoms, and readiness to quit using tobacco and nicotine were not associated with scores on any of the Reasons for Polytobacco Use Scale subscales. Parental use may influence use patterns less during this phase of life, particularly among college students who are immersed in college life and more influenced by peers ${ }^{32}$. Parental use may also be mediated by another more proximal variable. While depressive symptoms per say were not associated with the distinct subscale scores, using for affect regulation motives was, particularly with Displacement reasons for polytobacco use, which was also highly associated with nicotine dependence. This might potentially indicate that those using tobacco products during this transitory part of life to regulate negative emotions may be at risk for increased depressive symptoms later in life. Finally, the null findings for readiness to quit using tobacco or nicotine may reflect the phenomenon that many young adult users, particularly nondaily tobacco users, may not perceive themselves as "smokers" and thus not perceive a need to "quit" using ${ }^{53}$.

The current study has implications for research and practice. Future research might leverage these findings to develop cessation or prevention interventions that address the range of reasons for tobacco and polytobacco use in young adults. Additionally, examination of reasons for concurrent use of tobacco and marijuana might be important, given the high concurrent use rates of these products ${ }^{54,55}$. In practice, health educators and clinicians must be aware of the high polytobacco use rates among young adults and the different user profiles in order to effectively identify young adult polytobacco users and intervene to address these reasons for use.

\section{Limitations}

This study has some limitations. First, the study sample was drawn from colleges/universities in Georgia, is subject to selection bias, and may not generalize to all young adults. However, our sample is diverse in terms of race/ethnicity, geographic location (urban vs. rural), and socioeconomic backgrounds. Second, our scope of items may not be inclusive of all potentially important reasons for polytobacco use; however, the items developed and included here were drawn from the literature related to tobacco or polytobacco use in this population. Third, the cross-sectional design does not allow us to draw causal attributions or determine intra-individual trajectories of substance use over time. These analyses are also limited by the self-report nature of the assessments. Additionally, whether or not the participant was aware of the substance use of others (e.g., parents or friends) is a limitation of the data.

\section{CONCLUSIONS}

This study highlighted the prevalence of polytobacco use in young adults, as nearly half of all tobacco users used more than one tobacco product in the past 4 months, with nearly half of the polytobacco users using 4 or more different tobacco products. The current study addressed a gap in the literature, specifically regarding how to quantitatively assess and characterize reasons for polytobacco use. Four factors indicated distinct reasons for use: Instrumentality, Social Context, Displacement, and Experimentation. Each of these factors demonstrated unique convergent and discriminant validity. The use of this scale to characterize polytobacco using young adults may help inform and target interventions aimed at addressing tobacco and polytobacco use among young adults.

\section{REFERENCES}

1. Rigotti, N., J.E. Lee, and H. Wechsler, US college students' use of tobacco products: Results of a national survey. JAMA, 2000. 284: p. 699-705.

2. Smith-Simone, S., et al., Waterpipe tobacco smoking: knowledge, attitudes, beliefs, and behavior in two U.S. samples. Nicotine Tob Res, 2008. 10(2): p. 393-8. 
3. Knishkowy, B. and Y. Amitai, Water-pipe (narghile) smoking: an emerging health risk behavior. Pediatrics, 2005. 116(1): p. e113-9.

4. Etter, J.F., Electronic cigarettes: a survey of users. BMC Public Health, 2010. 10: p. 231.

5. Schoenborn, C.A. and R.M. Gindi, Electronic Cigarette Use Among Adults: United States, 2014. NCHS Data Brief, 2015. 217.

6. Hoffmann, D. and I. Hoffman, Chemistry and toxicology. Cigars: health effects and trends, in Smoking and Tobacco Control Monograph No. 9 1998, U.S. DHHS, National Institutes of Health, National Cancer Institute: Rockville, MD. p. 55-104.

7. Benowitz, N.L. and M.L. Goniewicz, The regulatory challenge of electronic cigarettes. JAMA, 2013. 310(7): p. 685-6.

8. Farsalinos, K.E. and R. Polosa, Safety evaluation and risk assessment of electronic cigarettes as tobacco cigarette substitutes: a systematic review. Ther Adv Drug Saf, 2014. 5(2): p. 67-86.

9. Bullen, C., et al., Electronic cigarettes for smoking cessation: a randomised controlled trial. Lancet, 2013. 382(9905): p. 1629-37.

10. Caponnetto, P., et al., EffiCiency and Safety of an eLectronic cigAreTte (ECLAT) as tobacco cigarettes substitute: a prospective 12-month randomized control design study. PLoS One, 2013. 8(6): p. e66317.

11. Berg, C.J., et al., Attitudes toward E-Cigarettes, Reasons for Initiating E-Cigarette Use, and Changes in Smoking Behavior after Initiation: A Pilot Longitudinal Study of Regular Cigarette Smokers. Open J Prev Med, 2014. 4(10): p. 789-800.

12. Food and Drug Administration, Summary of Results: Laboratory Analysis of Electronic Cigarettes Conducted By FDA. 2009, Food and Drug Administration: Silver Spring, MD.

13. Jensen, R.P., et al., Hidden formaldehyde in e-cigarette aerosols. N Engl J Med, 2015. 372(4): p. 392-4.

14. Grana, R., N. Benowitz, and S.A. Glantz, E-cigarettes: a scientific review. Circulation, 2014. 129(19): p. 1972-86.

15. Agaku, I.T., et al., Tobacco product use among adults--United States, 2012-2013. MMWR Morb Mortal Wkly Rep, 2014. 63(25): p. 5427.

16. Enofe, N., C.J. Berg, and E. Nehl, Alternative tobacco product use among college students: Who is at highest risk? Am J Health Behav, 2014. 38(2): p. 180-189.

17. Rath, J.M., et al., Patterns of tobacco use and dual use in US young adults: the missing link between youth prevention and adult cessation. J Environ Public Health, 2012. 2012: p. 679134.

18. Richardson, A., et al., The next generation of users: prevalence and longitudinal patterns of tobacco use among US young adults. Am J Public Health, 2014. 104(8): p. 1429-36.

19. Lee, Y.O., et al., Youth tobacco product use in the United States. Pediatrics, 2015. 135(3): p. 409-15.

20. Lee, Y.O., et al., Multiple tobacco product use among adults in the United States: cigarettes, cigars, electronic cigarettes, hookah, smokeless tobacco, and snus. Prev Med, 2014. 62: p. 14-9.

21. Soneji, S., J. Sargent, and S. Tanski, Multiple tobacco product use among US adolescents and young adults. Tob Control, 2016. 25(2): p. $174-80$.

22. Sung, H.Y., et al., Polytobacco Use of Cigarettes, Cigars, Chewing Tobacco, and Snuff Among US Adults. Nicotine Tob Res, 2016. 18(5): p. 817-26.

23. Tomar, S.L., H.R. Alpert, and G.N. Connolly, Patterns of dual use of cigarettes and smokeless tobacco among US males: findings from national surveys. Tob Control, 2010. 19(2): p. 104-9.

24. Fagerstrom, K. and T. Eissenberg, Dependence on tobacco and nicotine products: a case for product-specific assessment. Nicotine Tob Res, 2012. 14(11): p. 1382-90.
25. Etter, J.F. and T. Eissenberg, Dependence levels in users of electronic cigarettes, nicotine gums and tobacco cigarettes. Drug Alcohol Depend, 2015. 147: p. 68-75.

26. Ramo, D.E., H. Liu, and J.J. Prochaska, Validity and reliability of the nicotine and marijuana interaction expectancy (NAMIE) questionnaire. Drug Alcohol Depend, 2013. 131(1-2): p. 166-70.

27. Valjent, E., et al., Behavioural and biochemical evidence for interactions between Delta 9-tetrahydrocannabinol and nicotine. $\mathrm{Br}$ J Pharmacol, 2002. 135(2): p. 564-78.

28. Schauer, G.L., et al., Differences in the relationship of marijuana and tobacco by frequency of use: a qualitative study with young adults. Psychol Addict Behav, In press.

29. Martinasek, M.P., R.J. McDermott, and L. Martini, Waterpipe (hookah) tobacco smoking among youth. Curr Probl Pediatr Adolesc Health Care, 2011. 41(2): p. 34-57.

30. Klein, J.D., Hookahs and waterpipes: cultural tradition or addictive trap? J Adolesc Health, 2008. 42(5): p. 434-5.

31. Smith, J.R., et al., Increasing Hookah Use in California. Am J Public Health, 2011.

32. Berg, C.J., et al., Perceived harm, addictiveness, and social acceptability of tobacco products and marijuana among young adults: marijuana, hookah, and electronic cigarettes win. Subst Use Misuse, 2015. 50(1): p. 79-89.

33. Berg, C.J., et al., Cigarette users interest in using or switching to electronic nicotine delivery systems (ENDS) or smokeless tobacco for harm reduction, cessation, or novelty. Nicotine Tob Res, 2014.

34. Berg, C.J., Preferred flavors and reasons for e-cigarette use and discontinued use among never, current, and former smokers. Int J Public Health, 2016. 61(2): p. 225-36.

35. Montano, D.E. and D. Kasprzyk, The theory of reasoned action and the theory of planned behavior, in Health behavior and health education: Theory, research, and practice, K. Glanz, B.K. Rimer, and F.M. Lewis, Editors. 2002, Jossey-Bass: San Francisco. p. 67-98.

36. Ajzen, I., The theory of planned behavior. Organizational Behavior and Human Decision Processes, 1991. 50: p. 179-211.

37. Bandura, A., Social foundations of thought and action: A social cognitive theory. . 1986, Englewood Cliffs, NJ: Prentice Hall.

38. Stone, A.L., et al., Review of risk and protective factors of substance use and problem use in emerging adulthood. Addict Behav, 2012. 37(7): p. 747-75.

39. Hallfors, D.D., et al., Which comes first in adolescence--sex and drugs or depression? Am J Prev Med, 2005. 29(3): p. 163-70.

40. Windle, M. and M. Wiesner, Trajectories of marijuana use from adolescence to young adulthood: predictors and outcomes. Dev Psychopathol, 2004. 16(4): p. 1007-27.

41. Wu, L.T. and J.C. Anthony, Tobacco smoking and depressed mood in late childhood and early adolescence. Am J Public Health, 1999. 89(12): p. 1837-40.

42. Creswell, J.W. and V.L.P. Clark, Designing and Conducting Mixed Methods Research, 2nd Edition. 2011, Thousand Oaks, CA: Sage Publications.

43. Centers for Disease Control and Prevention, Behavioral Risk Factor Surveillance System, 2014. 2014, Centers for Disease Control and Prevention: Atlanta, GA.

44. Berg, C.J., et al., DECOY: Documenting Experiences with Cigarettes and Other Tobacco in Young Adults. American Journal of Health Behavior, 2016. 40(3): p. 310-321.

45. Wellman, R.J., et al., A comparison of the psychometric properties of the hooked on nicotine checklist and the modified Fagerstrom tolerance questionnaire. Addict Behav, 2006. 31(3): p. 486-95. 
46. Wellman, R.J., et al., Measuring adults' loss of autonomy over nicotine use: the Hooked on Nicotine Checklist. Nicotine Tob Res, 2005. 7(1): p. 157-61.

47. Piko, B.F., T.A. Wills, and C. Walker, Motives for smoking and drinking: Country and gender differences in samples of Hungarian and US high school students. Addict Behav, 2007. 32: p. 20872098.

48. Wills, T.A., J.M. Sandy, and O. Shinar, Cloninger's constructs related to substance use level and problems in late adolescence: a mediational model based on self-control and coping motives. Exp Clin Psychopharmacol, 1999. 7(2): p. 122-34.

49. Prochaska, J.O. and C.C. DiClemente, Self change processes, self-efficacy and decisional balance across five stages of smoking cessation, in Advances in Cancer Control-1983. 1984, Alan R. Liss, Inc: New York, NY. p. 131-140.

50. Kroenke, K., R.L. Spitzer, and J.B. Williams, The Patient Health Questionnaire-2: validity of a two-item depression screener. Medical care, 2003. 41(11): p. 1284-1292.

51. Haardörfer, R., et al., Polytobacco, marijuana, and alcohol use patterns in college students: A latent class analysis. Addict Behav, In press.

52. Evans-Polce, R., S. Lanza, and J. Maggs, Heterogeneity of alcohol, tobacco, and other substance use behaviors in U.S. college students: A latent class analysis. Addict Behav, 2016. 53: p. 80-5.

53. Berg, C.J., et al., Smoker self-identification versus recent smoking among college students. Am J Prev Med, 2009. 36(4): p. 333-6.

54. Schauer, G.L., et al., Assessing the overlap between tobacco and marijuana: Trends in patterns of co-use of tobacco and marijuana in adults from 2003-2012. Addict Behav, 2015. 49: p. 26-32.

55. Schauer, G.L., et al., Differences in Tobacco Product Use Among Past Month Adult Marijuana Users and Nonusers: Findings From the 2003-2012 National Survey on Drug Use and Health. Nicotine Tob Res, 2015.

ACKNOWLEDGMENTS

We would like to thank our Campus Advisory Board members across the state of Georgia in developing and assisting in administering this survey. We also would like to thank ICF Macro for their scientific input and technical support in conducting this research.

CONFLICT OF INTERESTS

All the authors have completed and submitted the ICMJE Form for Disclosure of Potential Conflicts of Interest and none were reported.

\section{FUNDING}

This research was supported by the National Cancer Institute (1RO1CA179422-01; PI: Berg). The funders had no role in the study design, collection, analysis or interpretation of the data, writing the manuscript, or the decision to submit the paper for publication.

PROVENANCE AND PEER REVIEW

Not commissioned;

Externally peer reviewed 